EPJ manuscript No.

(will be inserted by the editor)

\title{
Low- $x$ QCD physics from RHIC and HERA to the LHC
}

\author{
David d'Enterria ${ }^{1}$ \\ ${ }^{1}$ CERN, PH-EP, CH-1211 Geneva 23, Switzerland
}

Received: date / Revised version: date

\begin{abstract}
We present a summary of the physics of gluon saturation and non-linear QCD evolution at small values of parton momentum fraction $x$ in the proton and nucleus in the context of recent experimental results at HERA and RHIC. The rich physics potential of low- $x$ studies at the LHC, especially in the forward region, is discussed and some benchmark measurements in $p p, p A$ and $A A$ collisions are introduced.
\end{abstract}

PACS. 12.38.- $\mathrm{t}-24.85 .+\mathrm{p}-25.75 .-\mathrm{q}$

\section{Introduction}

\subsection{Parton structure and evolution}

The partonic structure of the proton (nucleus) can be probed with high precision in deep inelastic scattering (DIS) electronproton ep (electron-nucleus, $e A$ ) collisions. The inclusive DIS hadron cross section, $d^{2} \sigma / d x d Q^{2}$, is a function of the virtuality $Q^{2}$ of the exchanged gauge boson (i.e. its "resolving power"), and the Bjorken- $x$ fraction of the total nucleon momentum carried by the struck parton. The differential cross section for the neutral-current ( $\gamma, Z$ exchange) process can be written in terms of the target structure functions as

$$
\frac{d^{2} \sigma}{d x d Q^{2}}=\frac{2 \pi \alpha^{2}}{x Q^{4}}\left[Y_{+} \cdot F_{2} \mp Y_{-} \cdot x F_{3}-y^{2} \cdot F_{L}\right],
$$

where $Y_{ \pm}=1 \pm(1-y)^{2}$ is related to the collision inelasticity $y$, and the structure functions $F_{2,3, L}\left(x, Q^{2}\right)$ describe the density of quarks and gluons in the hadron: $F_{2} \propto e_{q}^{2} x \Sigma_{i}\left(q_{i}+\bar{q}_{i}\right), x F_{3} \propto$ $x \Sigma_{i}\left(q_{i}-\bar{q}_{i}\right), F_{L} \propto \alpha_{s} x g\left(x q_{i}, x \bar{q}_{i}\right.$ and $x g$ are the corresponding parton distribution functions, PDF). $F_{2}$, the dominant contribution to the cross section over most of phase space, is seen to rise strongly for decreasing Bjorken- $x$ at HERA (Fig. 1). The growth in $F_{2}$ is well described by $F_{2}\left(x, Q^{2}\right) \propto x^{-\lambda\left(Q^{2}\right)}$, with $\lambda \approx$ $0.1-0.3$ logarithmically rising with $Q^{2}[1]$. The $F_{2}$ scaling violations evident at small $x$ in Fig. 1 are indicative of the increasing gluon radiation from sea quarks. The $x g\left(x, Q^{2}\right)$ distribution itself can be indirectly determined (Fig. 2) from the $F_{2}$ slope:

$$
\frac{\partial F_{2}\left(x, Q^{2}\right)}{\partial \ln \left(Q^{2}\right)} \approx \frac{10 \alpha_{s}\left(Q^{2}\right)}{27 \pi} x g\left(x, Q^{2}\right) .
$$

Although the PDFs are non-perturbative objects obtained from fits to the DIS data, once measured at an input scale $Q_{0}^{2} \gtrsim$ $2 \mathrm{GeV}^{2}$ their value at any other $Q^{2}$ can be determined with the Dokshitzer-Gribov-Lipatov-Altarelli-Parisi (DGLAP) evolution equations which govern the probability of parton branchings (gluon splitting, $q, g$-strahlung) in QCD [3].

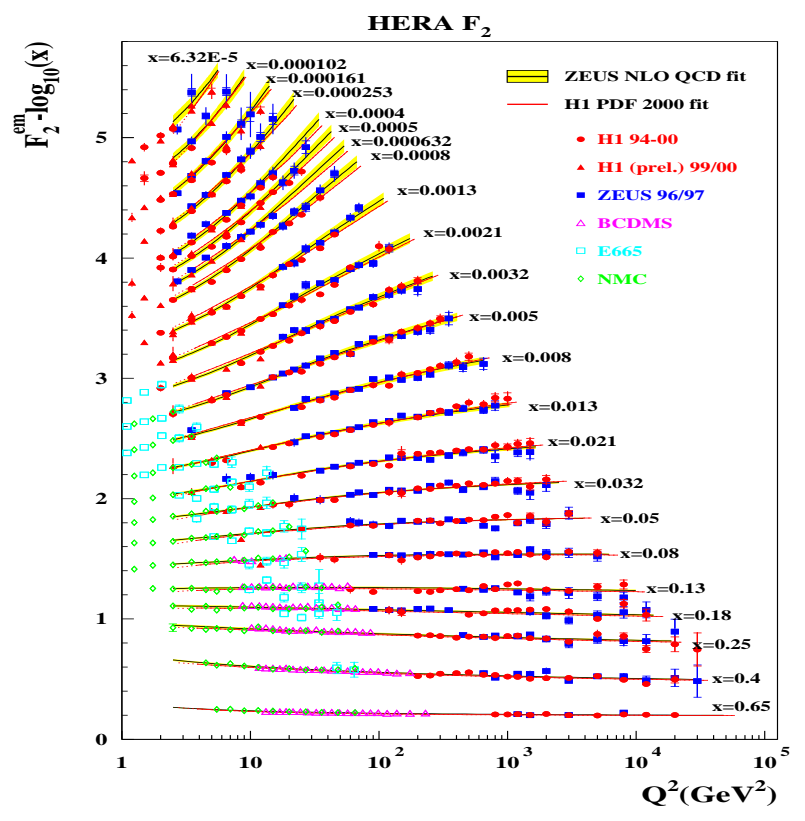

Fig. 1. $F_{2}\left(x, Q^{2}\right)$ measured in proton DIS at HERA $(\sqrt{s}=320 \mathrm{GeV})$ and fixed-target ( $\sqrt{s} \approx 10-30 \mathrm{GeV}$ ) experiments

The DGLAP parton evolution, however, only takes into account the $Q^{2}$-dependence of the PDFs, effectively summing leading powers of $\left[\alpha_{s} \ln \left(Q^{2}\right)\right]^{n}$ ("leading twist") generated by parton cascades in a region of phase space where the gluons have strongly-ordered transverse momenta towards the hard subcollision $Q^{2} \gg k_{n T}^{2} \gg \cdots \gg k_{1 T}^{2}$. Such a resummation is appropriate when $\ln \left(Q^{2}\right)$ is much larger than $\ln (1 / x)$. For decreasing $x$, the probability of emitting an extra gluon increases as $\propto \alpha_{s} \ln (1 / x)$. In this regime, the evolution of parton densities proceeds over a large rapidity region, $\Delta y \sim \ln (1 / x)$, and the finite transverse momenta of the partons become increasingly important. Here the full $k_{T}$ phase space of the gluons (including scattering of off-shell partons) has to be taken into ac- 


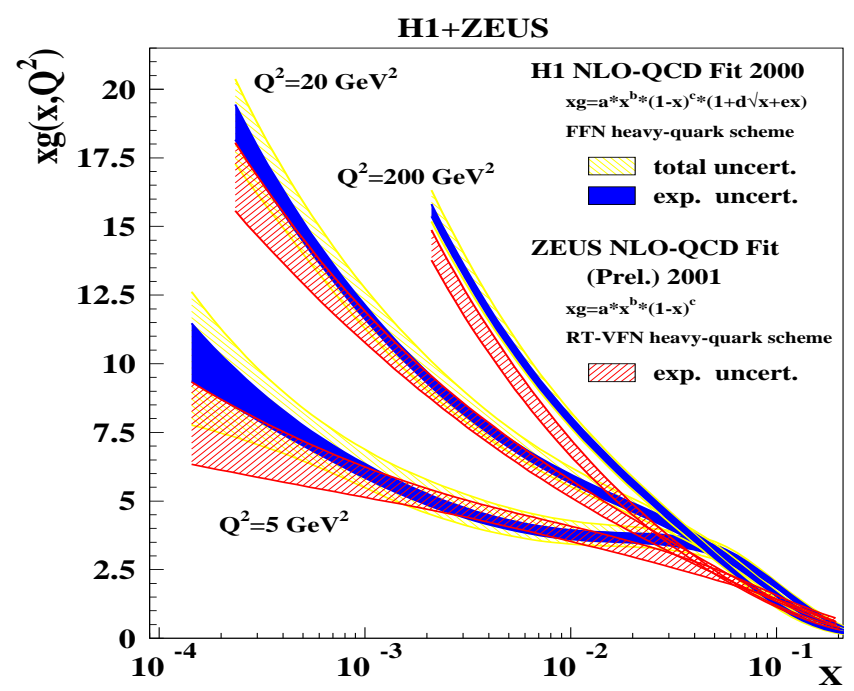

Fig. 2. Gluon distributions extracted at HERA (H1 and ZEUS) as a function of $x$ in three bins of $Q^{2}[2]$

count and not just the strongly-ordered DGLAP part. Thus, the appropriate description of the parton distributions is in terms of $k_{T}$-unintegrated PDFs, $x g\left(x, Q^{2}\right)=\int Q^{Q^{2}} d k_{T}^{2} G\left(x, k_{T}^{2}\right)$ with $G\left(x, k_{T}^{2}\right) \sim h\left(k_{T}^{2}\right) x^{-\lambda}$, described by the Balitski-Fadin-KuraevLipatov (BFKL) equation [4] which governs parton evolution in $x$ at fixed $Q^{2}$. Hints of extra BFKL radiation have been recently found at HERA in the enhanced production of forward jets compared to DGLAP expectations [5 6]. At large $Q^{2}$, a description resumming over both $\alpha_{s} \ln \left(Q^{2}\right)$ and $\alpha_{s} \ln (1 / x)$ is given by the Ciafaloni-Catani-Fiorani-Marchesini (CCFM) evolution equation [7].

\subsection{Parton saturation and non-linear evolution at low $x$}

As shown in Figure 2 the gluon density rises very fast for decreasing $x$. Eventually, at some small enough value of $x$ $\left(\alpha_{s} \ln (1 / x) \gg 1\right)$ the number of gluons is so large that nonlinear ( $g g$ fusion) effects become important, taming the growth of the parton densities. In such a high-gluon density regime three things are expected to occur: (i) the standard DGLAP and BFKL linear equations should no longer be applicable since they only account for single parton branchings $(1 \rightarrow 2$ processes) but not for non-linear $(2 \rightarrow 1)$ gluon recombinations; (ii) pQCD (collinear and $k_{T}$ ) factorization should break due to its (now invalid) assumption of incoherent parton scattering; and, as a result, (iii) standard $\mathrm{pQCD}$ calculations lead to a violation of unitarity even for $Q^{2} \gg \Lambda_{Q C D}^{2}$. Figure 3 schematically depicts the different parton evolution regimes as a function of $y=\ln (1 / x)$ and $Q^{2}$. For small enough $x$ values and for virtualities below an energy-dependent "saturation momentum", $Q_{s}$, intrinsic to the size of the hadron, one expects to enter the regime of saturated PDFs. Since $x g\left(x, Q^{2}\right)$ can be interpreted as the number of gluons with transverse area $r^{2} \sim 1 / Q^{2}$ in the hadron wavefunction, an increase of $Q^{2}$ effectively diminishes the 'size' of each parton, partially compensating for the growth in their number (i.e. the higher $Q^{2}$ is, the smaller the $x$ at which

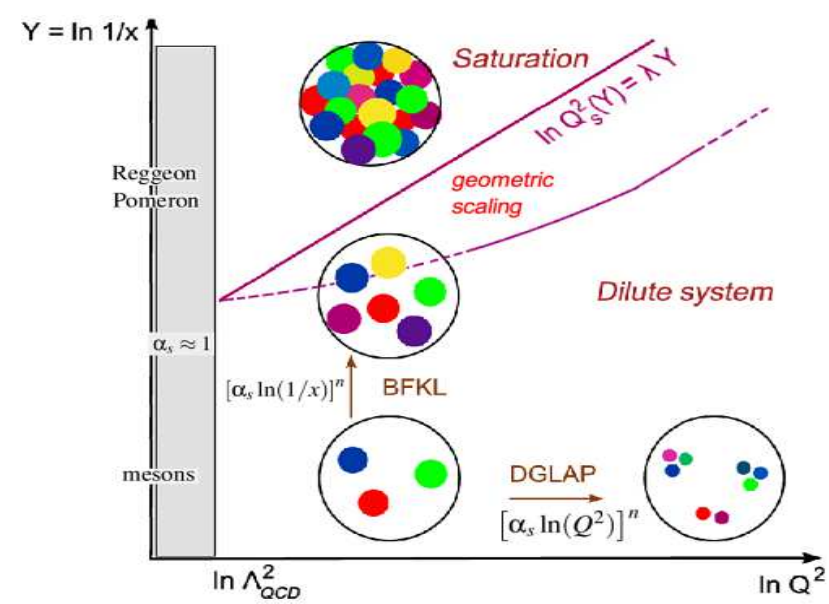

Fig. 3. QCD "phase diagram" in the $1 / x, Q^{2}$ plane (each dot represents a parton with transverse area $\sim 1 / Q^{2}$ and fraction $x$ of the hadron momentum). The different evolution regimes (DGLAP, BFKL, saturation) as well as the "saturation scale" and "geometric scaling" curves between the dense and dilute domains are indicated. Adapted from [8]

saturation sets in). Saturation effects are, thus, expected to occur when the size occupied by the partons becomes similar to the size of the hadron, $\pi R^{2}$. In the case of nuclear targets with $A$ nucleons (i.e. with gluon density $x G=A \cdot x g$ ), this condition provides a definition for the saturation scale [9, 10]:

$Q_{s}^{2}(x) \simeq \alpha_{s} \frac{1}{\pi R^{2}} x G\left(x, Q^{2}\right) \sim A^{1 / 3} x^{-\lambda} \sim A^{1 / 3}(\sqrt{s})^{\lambda} \sim A^{1 / 3} e^{\lambda y}$,

with $\lambda \approx 0.25$ [11]. Eq. (3) tell us that $Q_{s}$ grows with the number of nucleons in the target and with the energy of the collision, $\sqrt{s}$, or equivalently, the rapidity of the gluon $y=\ln (1 / x)$. The nucleon number dependence implies that, at equivalent energies, saturation effects will be enhanced by factors as large as $A^{1 / 3} \approx 6$ in heavy nuclear targets $(A=208$ for $P b)$ compared to protons. In the last fifteen years, an effective field theory of QCD in the high-energy (high density, small $x$ ) limit has been developed - the Colour Glass Condensate (CGC) [12] which describes the hadrons in terms of classical fields (saturated gluon wavefunctions) below the saturation scale $Q_{s}$. The saturation momentum $Q_{s}$ introduces a (semi-)hard scale, $Q_{s} \gg$ $\Lambda_{Q C D}$, which not only acts as an infrared cut-off to unitarize the cross sections but allows weak-coupling perturbative calculations $\left(\alpha_{s}\left(Q_{s}\right) \ll 1\right)$ in a strong $F_{\mu \nu}$ colour field background. In the CGC framework, hadronic and nuclear collisions are seen as collisions of classical wavefunctions which "resum" all gluon recombinations and multiple scatterings. The quantum evolution in the CGC approach is given by the JIMWLK [13] non-linear equations (or by their mean-field limit for $N_{c} \rightarrow \infty$, the Balitsky-Kovchegov equation [14]) which reduce to the standard BFKL kernel at higher $x$ values.

\section{Parton saturation: Experimental studies}

The main source of information on the PDFs is obtained from hard processes as they involve outgoing particles directly coupled to the partonic scattering vertices. Figure 4 summarizes 


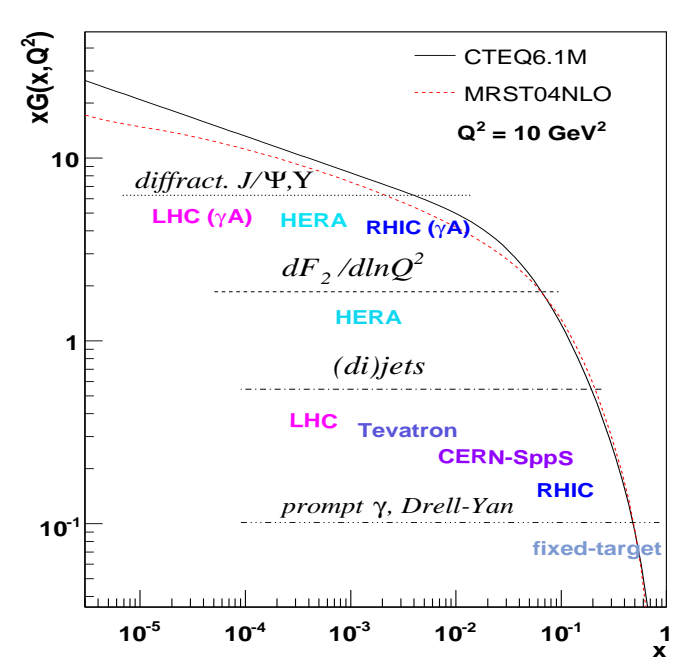

Fig. 4. Examples of experimental measurements at various facilities providing information on the gluon PDF in the range $x \sim 10^{-5}-0.8$

the variety of measurements at different experimental facilities which are sensitive to the gluon density and their approximate $x$ coverage. $x G$ enters directly at LO in hadron-hadron collisions with (i) prompt photons, (ii) jets, and (iii) heavy-quarks in the final state, as well as in the (difficult) DIS measurement ${ }^{1}$ of (iv) the longitudinal structure function $F_{L}$. In addition, (iv) heavy vector mesons $(J / \psi, \Upsilon)$ from diffractive photoproduction processes $^{2}$ are a valuable probe of the gluon density since their cross sections are proportional to the square of $x G$ [16 17]:

$$
\begin{array}{r}
\left.\frac{d \sigma_{\gamma p, A \rightarrow V p, A}}{d t}\right|_{t=0}=\frac{\alpha_{s}^{2} \Gamma_{e e}}{3 \alpha M_{V}^{5}} 16 \pi^{3}\left[x G\left(x, Q^{2}\right)\right]^{2} \\
\text { with } Q^{2}=M_{V}^{2} / 4 \text { and } x=M_{V}^{2} / W_{\gamma p, A}^{2} .
\end{array}
$$

The main source of information on the quark densities is obtained from measurements of (i) the structure functions $F_{2,3}$ in lepton-hadron scattering, and (ii) lepton pair (Drell-Yan, DY) production in hadron-hadron collisions. In hadronic collisions, one commonly measures the perturbative probes at central rapidities $(y=0)$ where $x=x_{T}=Q / \sqrt{s}$, and $Q \sim p_{T}, M$ is the characteristic scale of the hard scattering. However, one can probe smaller $x_{2}$ values in the target by measuring the corresponding cross sections in the forward direction. Indeed, for a $2 \rightarrow 2$ parton scattering the minimum momentum fraction probed in a process with a particle of momentum $p_{T}$ produced at pseudo-rapidity $\eta$ is 18

$$
x_{2}^{\min }=\frac{x_{T} e^{-\eta}}{2-x_{T} e^{\eta}} \quad \text { where } x_{T}=2 p_{T} / \sqrt{s},
$$

i.e. $x_{2}^{\min }$ decreases by a factor of $\sim 10$ every 2 units of rapidity. Though Eq. (6) is a lower limit at the end of phase-space (in

\footnotetext{
$1 x G$ can also be (in)directly extracted from $F_{2}$ through the derivative in Eq. 2] as well as from the $F_{2}^{\text {charm }}$ data [15].

2 Diffractive $\gamma p(\gamma A)$ processes are characterized by a quasi-elastic interaction - mediated by a Pomeron or two gluons in a colour singlet state - in which the $p(A)$ remains intact (or in a low excited state) and separated by a rapidity gap from the rest of final-state particles.
}

practice the $\left\langle x_{2}\right\rangle$ values in parton-parton scatterings are at least 10 larger than $x_{2}^{\min }[18]$ ), it provides the right estimate of the typical $x_{2}=\left(p_{T} / \sqrt{s}\right) e^{-\eta}$ values reached in non-linear $2 \rightarrow 1$ processes (in which the momentum is balanced by the gluon "medium") as described in parton saturation models [19 20].

Figure 5 shows the kinematical map in $\left(x, Q^{2}\right)$ of the existing DIS, DY, direct $\gamma$ and jet data used in the PDF fits. Results from HERA and the Tevatron cover a substantial range of the proton structure $\left(10^{-4} \lesssim x \lesssim 0.8,1 \lesssim Q^{2} \lesssim 10^{5} \mathrm{GeV}^{2}\right)$ but the available measurements are much rarer in the case of nuclear targets (basically limited to fixed-target studies, $10^{-2} \lesssim x \lesssim 0.8$ and $1 \lesssim Q^{2} \lesssim 10^{2} \mathrm{GeV}^{2}$ ). As a matter of fact, the nuclear parton distributions are basically unknown at low $x(x<0.01)$ where the only available measurements are fixed-target data in the non-perturbative range $\left(Q^{2}<1 \mathrm{GeV}^{2}\right)$ dominated by Regge dynamics rather than quark/gluon degrees of freedom. An example of the current lack of knowledge of the nuclear densities at low $x$ is presented in Fig. 6 where different available parametrizations of the ratio of $P b$ to proton gluon distributions, consistent with the available nDIS data at higher $x$, show differences as large as a factor of three at $x \sim 10^{-4}[2324]$.

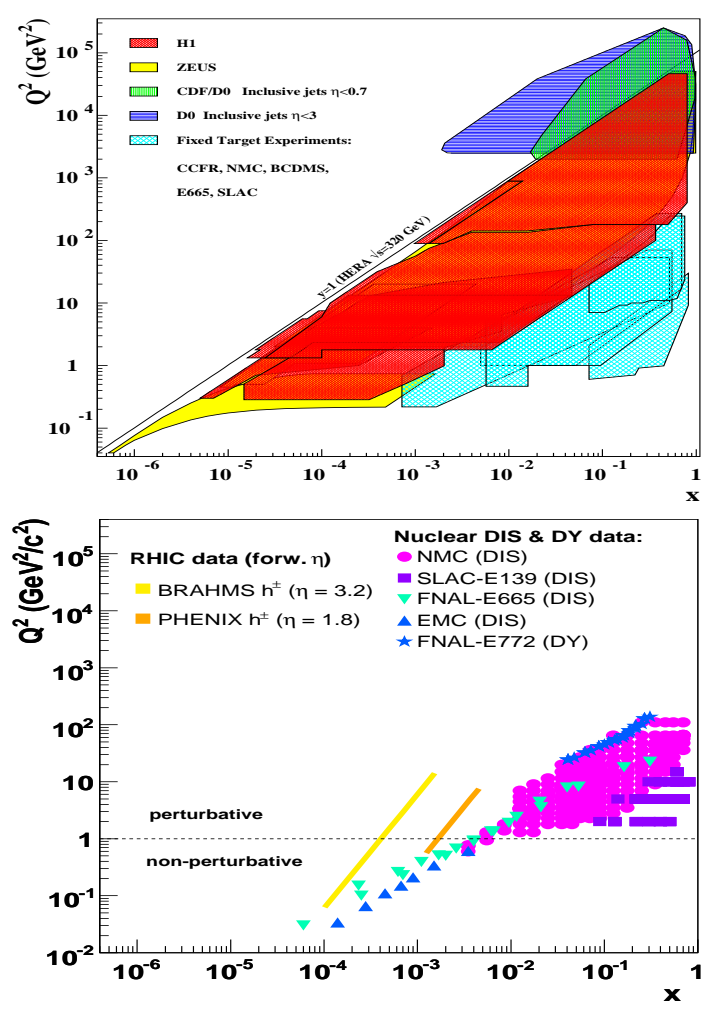

Fig. 5. Available measurements in the $\left(x, Q^{2}\right)$ plane used for the determination of the proton [21] (top) and nuclear [22] (bottom) PDFs

\subsection{HERA results}

Though the large majority of ep DIS data collected during the HERA-I phase are successfully reproduced by standard DGLAP predictions, more detailed and advanced experimental and theoretical results in the recent years have pointed to 


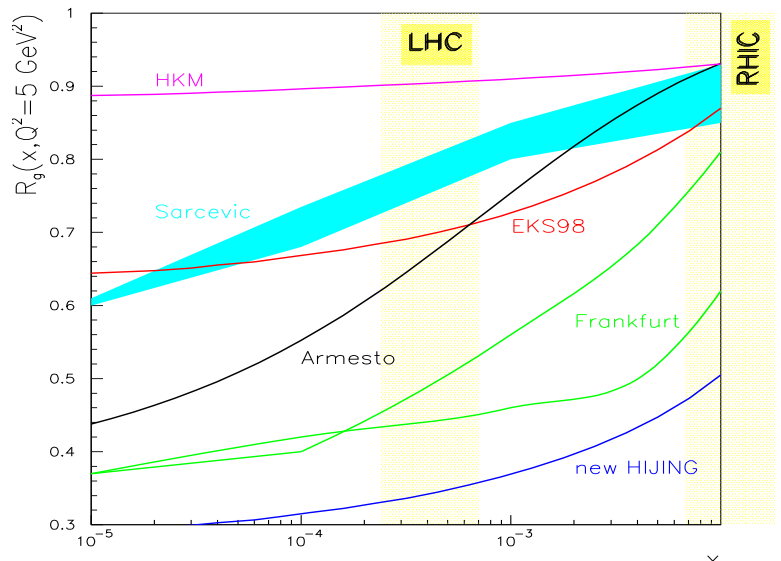

Fig. 6. Ratios of the $P b$ over proton gluon PDFs versus $x$ from different models at $Q^{2}=5 \mathrm{GeV}^{2}$. Figure taken from 23

interesting hints of non-linear QCD effects in the data. Arguably, the strongest indication of such effects is given by the so-called "geometric scaling" property observed in inclusive $\sigma_{D I S}$ for $x<0.01[25]$ as well as in various diffractive cross sections [26 27]. For inclusive DIS events, this feature manifests itself in a total cross section at small $x(x<0.01)$ which is only a function of $\tau=Q^{2} / Q_{s}^{2}(x)$, instead of being a function of $x$ and $Q^{2} / Q_{s}^{2}$ separately (Fig. [7). The saturation momentum follows $Q_{s}(x)=Q_{0}\left(x / x_{0}\right)^{\lambda}$ with parameters $\lambda \sim 0.3, Q_{0}=1 \mathrm{GeV}$, and $x_{0} \sim 3 \cdot 10^{-4}$. Interestingly, the scaling is valid up to very large values of $\tau$, well above the saturation scale, in an "extended scaling" region (see Fig. 3] where $Q_{s}^{2}<Q^{2}<Q_{s}^{4} / \Lambda_{O C D}^{2}[28$, 8]. The saturation formulation is suitable to describe not only inclusive DIS, but also inclusive diffraction $\gamma^{\star} p \rightarrow X p$. The very similar energy dependence of the inclusive diffractive and total cross sections in $\gamma^{\star} p$ collisions at a given $Q^{2}$ is easily explained in the Golec-Biernat Wüsthoff model [25] but not in standard collinear factorization. Furthermore, geometric scaling has been also found in different diffractive DIS cross sections (inclusive, vector mesons, deeply-virtual Compton scattering DVCS) [26, 27]. All the observed scalings are suggestive manifestations of the QCD saturation regime. Unfortunately, the values of $Q_{s}^{2} \sim 0.5-1.0 \mathrm{GeV}$ at HERA lie in the transition region between the soft and hard sectors and, therefore, non-perturbative effects obscure the obtention of clearcut experimental signatures.

\subsection{RHIC results}

The expectation of enhanced parton saturation effects in the $\mathrm{nu}$ clear wave functions accelerated at ultra-relativistic energies, Eq. (3), has been one of the primary physics motivations for the heavy-ion programme at RHIC ${ }^{3}[12]$. Furthermore, the properties of the high-density matter produced in the final-state of $A A$ interactions cannot be properly interpreted without having isolated first the influence of initial state modifications of the

${ }^{3}$ At $y=0$, the saturation scale for a $A u$ nucleus at RHIC $\left(Q_{s}^{2} \sim 2\right.$ $\mathrm{GeV})$ is larger than that of protons probed at HERA $\left(Q_{s}^{2} \sim 0.5 \mathrm{GeV}\right)$.

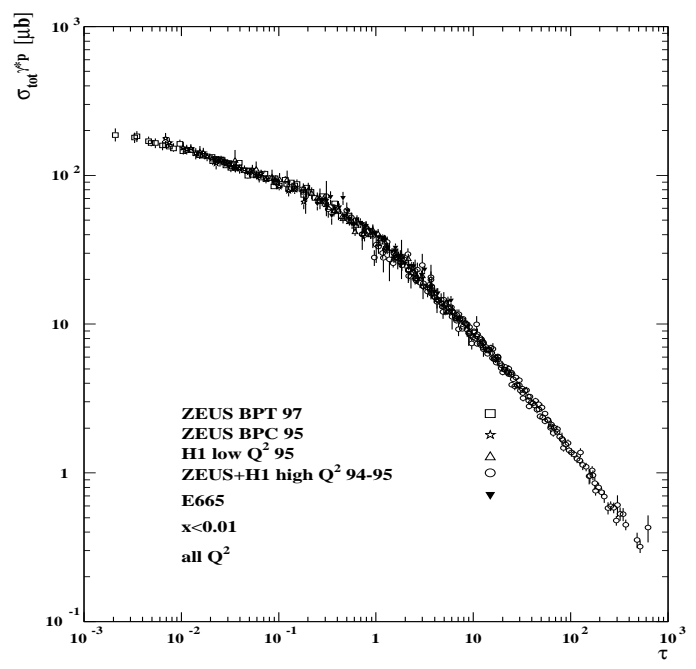

Fig. 7. Geometric scaling in the DIS $\gamma^{\star} p$ cross sections plotted versus $\tau=Q^{2} / Q_{s}^{2}$ in the range $x<0.01,0.045<Q^{2}<450 \mathrm{GeV}^{2}$ [25]

nuclear PDFs. After five years of operation, two main experimental results at RHIC have been found consistent with CGC predictions: (i) the modest hadron multiplicities measured in $A u A u$ reactions, and (ii) the suppressed hadron yield observed at forward rapidities in $d A u$ collisions. [In addition, a recent analysis of the existing nuclear DIS $F_{2}$ data also confirms the existence of "geometrical scaling" for $x<0.017$ [29].]

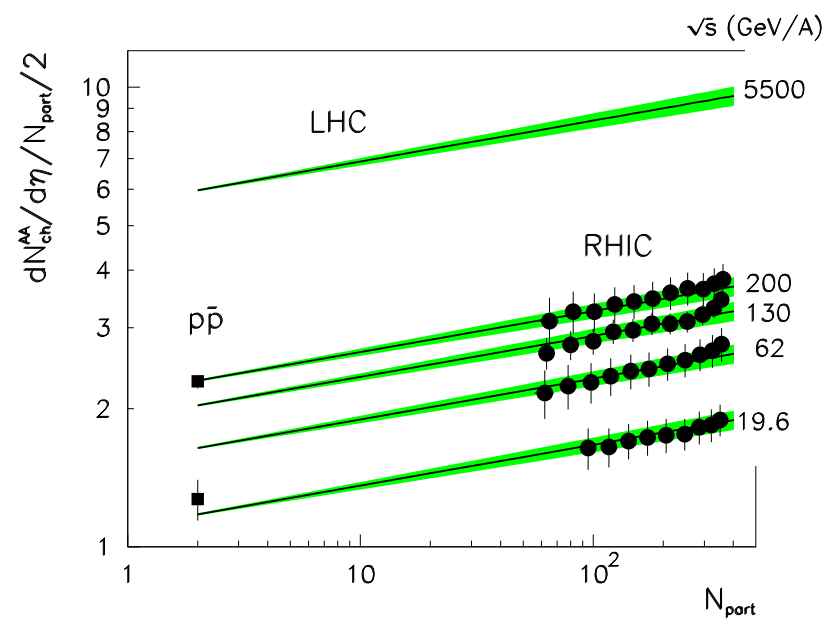

Fig. 8. Dependences on c.m. energy and centrality (given in terms of the number of nucleons participating in the collision, $N_{\text {part }}$ ) of $d N_{c h} /\left.d \eta\right|_{\eta=0}$ (normalized by $N_{\text {part }}$ ): PHOBOS $A u A u$ data [34 vs the predictions of the saturation approach [29]

The bulk hadron multiplicities measured at mid-rapidity in central $A u A u$ at $\sqrt{s_{N N}}=200 \mathrm{GeV}$ are $d N_{c h} /\left.d \eta\right|_{\eta=0} \approx 700$, comparatively lower than the $d N_{c h} /\left.d \eta\right|_{\eta=0} \approx 1000$ expectations of "minijet" dominated scenarios [30], soft Regge models [31] (without accounting for strong shadowing effects [32]), or extrapolations from an incoherent sum of proton-proton collisions [33]. On the other hand, CGC approaches [11,29] which effectively take into account a reduced number of scattering 
centers in the nuclear PDFs, $f_{a / A}\left(x, Q^{2}\right)<A \cdot f_{a / N}\left(x, Q^{2}\right)$ reproduce well not only the measured hadron multiplicities but based on the general expression (3) - also the centrality and c.m. energy dependences of the bulk $A A$ hadron production (Fig. 8).

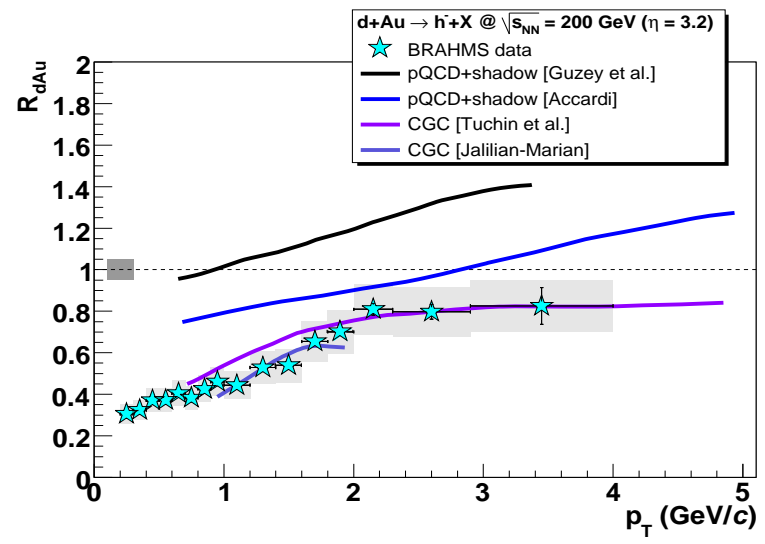

Fig. 9. Nuclear modification factor $R_{d A u}\left(p_{T}\right)$ for negative hadrons at $\eta=3.2$ in $d A u$ at $\sqrt{s_{N N}}=200 \mathrm{GeV}$ : BRAHMS data [35] compared to leading-twist shadowing pQCD [18 19] and CGC [36 37] predictions

The second manifestation of saturation-like effects in the RHIC data is the BRAHMS observation [35] of suppressed yields of moderately high- $p_{T}$ hadrons $\left(p_{T} \approx 2-4 \mathrm{GeV} / c\right)$ in $d A u$ relative to $p p$ collisions at $\eta \approx 3.2$ (Fig. 9). Hadron production at such small angles is sensitive to partons in the $A u$ nucleus with $x_{2} \approx \mathscr{O}\left(10^{-3}\right)$. The observed nuclear modification factor, $R_{d A u} \approx 0.8$, cannot be reproduced by $\mathrm{pQCD}$ calculations that include standard leading-twist shadowing of the nuclear PDFs [18 19] but can be described by CGC approaches [36 37] that parametrize the $A u$ nucleus as a saturated gluon wavefunction. As in the HERA case, it is worth noting however that at RHIC energies the saturation scale is in the transition between the soft and hard regimes $\left(Q_{s}^{2} \approx 2 \mathrm{GeV}^{2}\right)$ and the results consistent with the CGC predictions are in a kinematic range with relatively low momentum scales $\left(\left\langle p_{T}\right\rangle \sim 0.5 \mathrm{GeV}\right.$ for the hadron multiplicities and $\left\langle p_{T}\right\rangle \sim 2.5 \mathrm{GeV}$ for forward inclusive hadron production) where non-perturbative effects can blur a simple interpretation based on partonic degrees of freedom alone.

\section{Low- $x$ QCD at the LHC}

The Large Hadron Collider (LHC) at CERN will provide $p p$, $p A$ and $A A$ collisions at $\sqrt{s_{N N}}=14,8.8$ and $5.5 \mathrm{TeV}$ respectively with luminosities $\mathscr{L} \sim 10^{34}, 10^{29}$ and $5 \cdot 10^{26} \mathrm{~cm}^{-2} \mathrm{~s}^{-1}$. Such large c.m. energies and luminosities will allow detailed QCD studies at unprecedented low $x$ values thanks to the copious production of hard probes (jets, quarkonia, heavy-quarks, prompt $\gamma$, Drell-Yan pairs, etc.). The advance in the study of low- $x$ QCD phenomena will be specially substantial for nuclear systems since the saturation momentum, Eq. (3), $Q_{s}^{2} \approx 5-10$ $\mathrm{GeV}^{2}$, will be in the perturbative range [11], and the relevant $x$ values, Eq. (6), will be 30-70 times lower than $A A$ and $p A$ reactions at RHIC: $x \approx 10^{-3}\left(10^{-5}\right)$ at central (forward) rapidities for processes with $Q^{2} \sim 10 \mathrm{GeV}^{2}$ (Fig. [10).

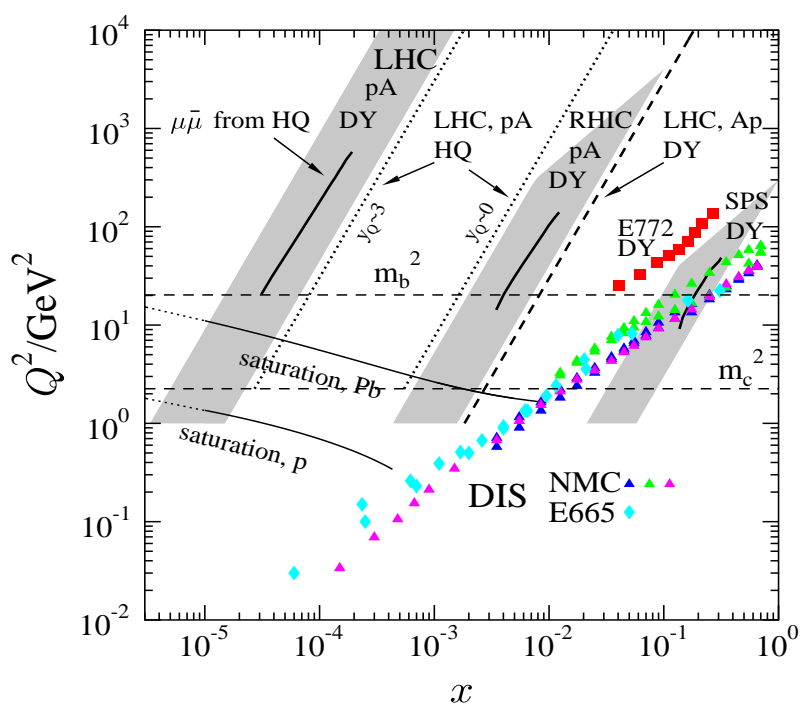

Fig. 10. Kinematical $\left(x, Q^{2}\right)$ range probed at various rapidities $y$ and c.m. energies in $\sqrt{s_{N N}}=8.8 \mathrm{TeV} p A$ collisions at the LHC [23]

\subsection{The LHC experiments}

The four LHC experiments - i.e. the two large general-purpose and high-luminosity ATLAS and CMS detector systems as well as the heavy-ion-dedicated ALICE and the heavy-flavouroriented LHCb experiments - have all detection capabilities in the forward direction very well adapted for the study of low- $x$ QCD phenomena with hard processes in collisions with proton and ion beams:

(i) Both CMS and ATLAS feature hadronic calorimeters in the range $3<|\eta|<5$ which allow them to measure jet crosssections at very forward rapidities. Both experiments feature also zero-degree calorimeters (ZDC, $|\eta| \gtrsim 8.5$ for neutrals), which are a basic tool for neutron-tagging "ultraperipheral" $\mathrm{PbPb}$ photoproduction interactions. CMS has an additional electromagnetic/hadronic calorimeter (CASTOR, $5.3<|\eta|<6.7)$ and shares the interaction point with the TOTEM experiment providing two extra trackers at very forward rapidities $(\mathrm{T} 1,3.1<|\eta|<4.7$, and $\mathrm{T} 2,5.5<$ $|\eta|<6.6)$ well-suited for DY measurements.

(ii) The ALICE forward muon spectrometer at $2.5<\eta<4$, can reconstruct $J / \psi$ and $\Upsilon$ (as well as $Z$ ) in the dimuon channel, as well as statistically measure single inclusive heavy-quark production via semileptonic (muon) decays. ALICE counts also on ZDCs in both sides of the interaction point (IP) for forward neutron triggering of $\mathrm{PbPb}$ photoproduction processes.

(iii) LHCb is a single-arm spectrometer covering rapidities $1.8<\eta<4.9$, with very good particle identification capabilities designed to accurately reconstruct $b$ and $c$ mesons. The detector is also well-suited to measure jets, $Q \bar{Q}$ and $\mathrm{Z} \rightarrow \mu \mu$ production in the forward hemisphere.

\subsection{Low- $x$ QCD measurements at LHC}

Measurement at the LHC forward rapidities of any of the processes shown in Fig. 4 4 provides an excellent means to look for 
signatures of high gluon density phenomena at low $x$. Four representative measurements are discussed in the last section of the paper.

- Case study I: Forward (di)jets ( $p p, p A, A A)$

The jet measurements in $\bar{p} p$ collisions at Tevatron energies have provide valuable information on the proton PDFs (see Fig. 5, top). According to Eq. (6), the measurement of jets with $p_{T} \approx 20-200 \mathrm{GeV} / c$ in $p p$ collisions at $14 \mathrm{TeV}$ in the ATLAS or CMS forward calorimeters $(3<|\eta|<5)$ allows one to probe the PDFs at $x$ values as low as $x_{2} \approx 10^{-4}-10^{-5}$. Fig. 11 shows the actual $\log \left(x_{1,2}\right)$ distribution of two partons scattering at LHC and producing at least one forward jet as obtained with PYTHIA 6.403 [38]. As expected in forward scattering, the collision is very asymmetric with $x_{2}\left(x_{1}\right)$ peaked at $\sim 10^{-4}$ $\left(\sim 10^{-1}\right)$ and thus provides direct information on the low- $x$ parton densities. Not only the single inclusive cross-section but the forward-backward dijet production, "Müller-Navelet jets", is a particularly sensitive measure of BFKL [39] as well as nonlinear [6] parton evolutions. In the presence of low- $x$ saturation effects, the Müller-Navelet cross section for two jets separated by $\Delta \eta \sim 9$ (and, thus, measurable in each one of the forward calorimeters) is expected to be suppressed by a factor of $\sim 2$ compared to BFKL predictions [6]. A study is underway to determine the feasibility of both forward (di)jet measurements in CMS [40].

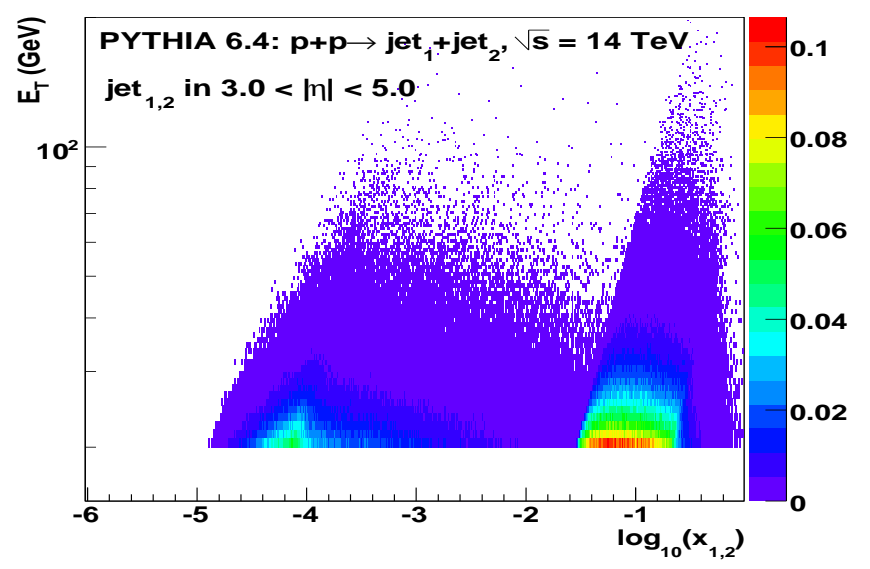

Fig. 11. $\log \left(x_{1,2}\right)$ distribution of two partons colliding in $p p$ collisions at $\sqrt{s}=14 \mathrm{TeV}$ and producing at least one jet within ATLAS/CMS forward calorimeters acceptances as determined with PYTHIA

\section{- Case study II: Forward heavy-quarks ( $p p, p A, A A)$}

Studies of small- $x$ effects on heavy flavour production at the LHC in two different approaches, based on collinear and $k_{T}$ factorization, including non-linear terms in the parton evolution, lead to two different predictions (enhancement vs. suppression) for the measured $c$ and $b$ cross-sections [15]. The possibility of ALICE and $\mathrm{LHCb}$ to reconstruct $D$ and $B$ mesons in a large rapidity range (Fig. 12) will put stringent constraints on the gluon structure and evolution at low- $x$. In the case of ALICE, the heavy-Q $p p$ studies will have a natural extension in $A A$ and $p A$ collisions [41], providing a precise probe of nonlinear effects in the nuclear wave-function.

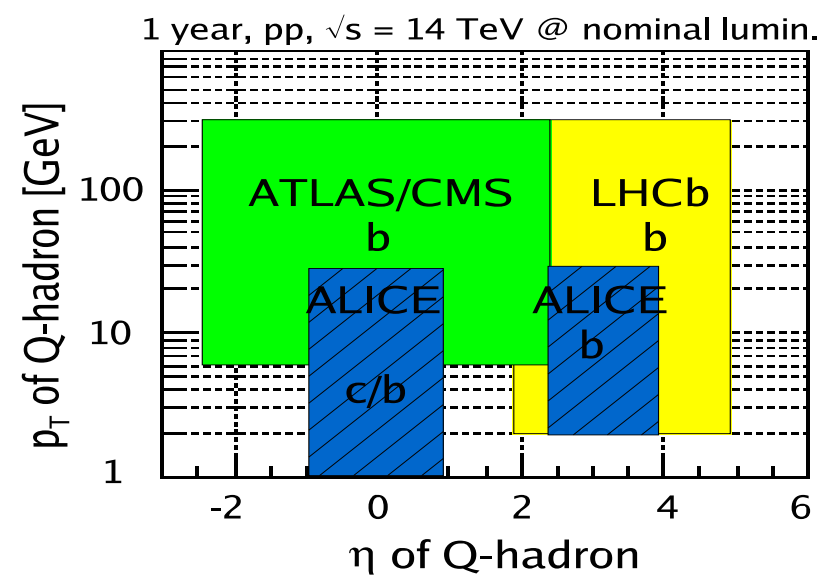

Fig. 12. Acceptances in $\left(\eta, p_{T}\right)$ for open charm and bottom hadrons in the four LHC experiments for 1-year nominal luminosity [15]

\section{- Case study III: $Q \bar{Q}$ photoproduction (electromagnetic $A A$ )}

High-energy diffractive photoproduction of heavy vector mesons $(J / \psi, \Upsilon)$ proceeds through colourless two-gluon exchange (which subsequently couples to $\gamma \rightarrow Q \bar{Q}$ ) and is thus a sensitive probe of the gluon densities at small $x$, see Eq. (4). Ultra-peripheral interactions (UPCs) of high-energy heavy ions generate strong electromagnetic fields which help constrain the low- $x$ behaviour of $x G$ via quarkonia [42], or other hard probes [43], produced in $\gamma$-nucleus collisions. Lead beams at 2.75 TeV have Lorentz factors $\gamma=2930$ leading to maximum (equivalent) photon energies $\omega_{\max } \approx \gamma / R \sim 100 \mathrm{GeV}$, and c.m. energies $W_{\gamma \gamma}^{\max } \approx 160 \mathrm{GeV}$ and $W_{\gamma A}^{\max } \approx 1 \mathrm{TeV}$. From Eq. (5), the $x$ values probed in $\gamma A \rightarrow J / \psi A$ processes at $y=2$ can be as low as $x \sim 10^{-5}$. The ALICE, ATLAS and CMS experiment can measure $J / \psi, \Upsilon \rightarrow e^{+} e^{-}, \mu^{+} \mu^{-}$produced in electromagnetic $\mathrm{PbPb}$ collisions tagged with neutrons detected in the ZDCs (as done at RHIC [42]). Figure 13 shows the expected dimuon invariant mass distributions around the $\Upsilon$ mass predicted by STARLIGHT [4] within the CMS central acceptance $(\eta<2.5)$ for an integrated $\mathrm{PbPb}$ luminosity of $0.5 \mathrm{nb}^{-1}[45]$.

\section{- Case study IV: Forward Drell-Yan pairs ( $p p, p A, A A)$}

High-mass Drell-Yan pair production at the very forward rapidities covered by $\mathrm{LHCb}$ and by the CMS CASTOR and TOTEM T2 detectors can probe the parton densities down to $x \sim 10^{-6}$ at much higher virtualities $M^{2}$ than those accessible in other measurements discussed here. A study is currently underway in CMS [40] to combine the CASTOR electromagnetic energy measurement together with the good position resolution of T2 for charged tracks, to trigger on and reconstruct the $e^{+} e^{-}$ invariant mass in $p p$ collisions at $14 \mathrm{TeV}$, and scrutinize $x g$ in the $M^{2}$ and $x$ plane. 


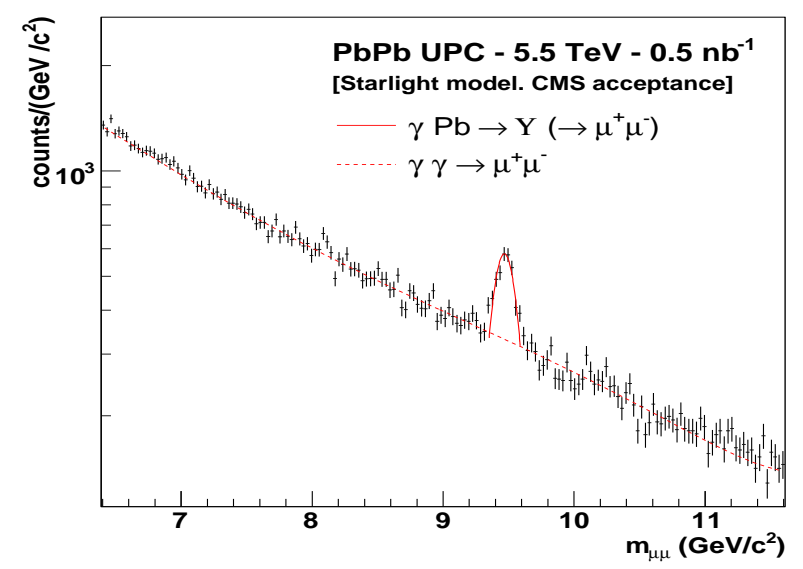

Fig. 13. Expected $\mu^{+} \mu^{-}$invariant mass from $\gamma P b \rightarrow \Upsilon P b^{\star} \rightarrow$ $\mu^{+} \mu^{-} P b^{\star}$ and $\gamma \gamma \rightarrow \mu^{+} \mu^{-}$processes predicted by Starlight [44] for UPC $P b P b$ collisions at $\sqrt{s_{N N}}=5.5 \mathrm{TeV}$ in the CMS acceptance

\section{Conclusion}

We have reviewed the physics of non-linear QCD and high gluon densities at small fractional momenta $x$, with emphasis on the existing data at HERA (proton) and RHIC (nucleus) which support the existence of a parton saturation regime as described e.g. in the framework of the Colour Glass Condensate effective field theory. The future perspectives at the LHC have been presented, including the promising capabilities of the forward detectors of the ALICE, ATLAS, CMS and LHCb experiments to study the parton densities down to $x \sim 10^{-6}$ with various hard probes (jets, quarkonia, heavy-quarks, Drell-Yan). The programme of investigating the dynamics of low- $x$ QCD is not only appealing in its own right but it is an essential prerequisite for predicting a large variety of hadron-, photon- and neutrino- scattering cross sections at very high energies.

\section{Acknowledgments}

The author thanks A. de Roeck and H. Jung as well as A. Dobado and F. J. Llanes Estrada for their invitation to present this overview talk in the 2nd HERA-LHC Workshop and in the QNP'06 Int. Conf. This work is supported by the 6th EU Framework Programme contract MEIF-CT-2005-025073.

\section{References}

1. C. Adloff et al. [H1 Collab.], Phys. Lett. B 520, 183 (2001)

2. M. Dittmar et al., in Proceeds. HERA-LHC Workshop, hep-ph/0511119

3. V.N. Gribov and L.N. Lipatov, Sov. Journ. Nucl. Phys. 15, 438 (1972); G. Altarelli and G. Parisi, Nucl. Phys. B126, 298 (1977); Yu. L. Dokshitzer, Sov. Phys. JETP 46, 641 (1977)

4. L.N. Lipatov, Sov. J. Nucl. Phys. 23, 338 (1976); E.A. Kuraev, L.N. Lipatov and V.S. Fadin, Zh. Eksp. Teor. Fiz 72, 3 (1977); Ya.Ya. Balitsky, L.N. Lipatov, Sov. J. Nucl. Phys. 28, (1978) 822
5. ZEUS Collaboration, S. Chekanov et al., Phys. Lett. B 632, 13 (2006); H1 Collaboration A. Aktas et al., Eur. Phys. J. C 46, 27 (2006)

6. C. Marquet and C. Royon, Nucl. Phys. B 739, 131 (2006)

7. S. Catani, F. Fiorani and G. Marchesini, Nucl. Phys. B 336, 18 (1990); S. Catani, M. Ciafaloni and F. Hautmann, Nucl. Phys. B 366, 135 (1991)

8. E. Iancu, C. Marquet and G. Soyez, hep-ph/0605174

9. L. Gribov, E. M. Levin and M. G. Ryskin, Phys. Rept. 1001 (1983)

10. A. H. Mueller and J. w. Qiu, Nucl. Phys. B 268427 (1986)

11. D. Kharzeev and M. Nardi, Phys. Lett. B 507, 121 (2001);

D. Kharzeev, E. Levin and M. Nardi, Nucl. Phys. A 747, 609 (2005)

12. See e.g. E. Iancu and R. Venugopalan, in QGP. Vol 3, Eds: R.C. Hwa et al., World Scientific, Singapore, hep-ph/0303204 J. JalilianMarian and Y. V. Kovchegov, Prog. Part. Nucl. Phys. 56, 104 (2006); and refs. therein

13. J. Jalilian-Marian, A. Kovner, A. Leonidov and H. Weigert, Nucl. Phys. B504, 415 (1997); Phys. Rev. D59, 014014 (1999); E. Iancu, A. Leonidov and L. McLerran, Nucl. Phys. A692, 583 (2001);

14. I. Balitsky, Nucl. Phys. B 463, 99 (1996) ; Yu.V. Kovchegov, Phys. Rev. D 61, 074018 (2000).

15. J. Baines et al., in Proceeds. HERA-LHC Workshop, hep-ph/0601164

16. M. G. Ryskin et al., Z. Phys. C76, 231 (1997)

17. T. Teubner, Proceeds. DIS'05, AIP Conf. Proc. 792, 416 (2006).

18. V. Guzey, M. Strikman, and W. Vogelsang, Phys. Lett. B 603, 173 (2004)

19. A. Accardi, Acta Phys.Hung. A22, 289 (2005)

20. A. Dumitru, A. Hayashigaki and J. Jalilian-Marian, Nucl. Phys. A 765, 464 (2006)

21. P. Newman, Int. J. Mod. Phys. A 19, 1061 (2004)

22. D. d'Enterria, J. Phys. G 30, S767 (2004)

23. A. Accardi et al., in CERN Yellow report Hard probes in Heavy Ion collisions at the LHC, hep-ph/0308248

24. N. Armesto, J. Phys. G32, R367 (2006)

25. K. Golec-Biernat and M. Wüsthoff, Phys. Rev. D59, 014017 (1999); Phys. Rev. D60, 114023 (1999)

26. J. R. Forshaw and G. Shaw, JHEP 0412052 (2004)

27. C. Marquet, L. Schoeffel, hep-ph/0606079

28. E. Iancu, K. Itakura, L. McLerran, Nucl. Phys. A708 327 (2002)

29. N. Armesto, C. A. Salgado and U. A. Wiedemann, Phys. Rev. Lett. 94, 022002 (2005)

30. HIIJING: M. Gyulassy and X.N. Wang, Comput. Phys. Commun. 83, 307 (1994)

31. DPM: A. Capella, U. Sukhatme, C.I. Tan and Van J. Tran Thanh, Phys. Rept. 236, 225 (1994)

32. N. Armesto and C. Pajares, Int. J. Mod. Phys. A 15, 2019 (2000)

33. K. J. Eskola, Nucl. Phys. A 698, 78 (2002)

34. PHOBOS, B. B. Back et al., Nucl. Phys. A757, 28 (2005)

35. BRAHMS, I. Arsene et al., Nucl. Phys. A757, 1 (2005)

36. D.Kharzeev, Y.Kovchegov, K.Tuchin, Phys. Lett. B599, 23 (2004)

37. J. Jalilian-Marian, Nucl. Phys. A 748, 664 (2005)

38. T. Sjostrand, S. Mrenna and P. Skands, JHEP 0605, 026 (2006)

39. A. H. Mueller and H. Navelet, Nucl. Phys. B 282, 727 (1987)

40. [CMS and TOTEM] "Prospects for Diffractive and Forward Physics at LHC', in preparation.

41. A. Dainese, Proceeds. Hard Probes'06, nucl-ex/0609042

42. D. d'Enterria, Proceeds. Quark Matter'05, nucl-ex/0601001

43. M. Strikman, R. Vogt and S. White, Phys. Rev. Lett. 96, 082001 (2006)

44. S. R. Klein, J. Nystrand, Phys. Rev. C60, 014903 (1999); A. Baltz, S. Klein, J. Nystrand, Phys. Rev. Lett. 89, 012301 (2002). 45. D. d'Enterria [CMS Collaboration], Eur. Phys. J. C to appear, hep-ex/0609019 